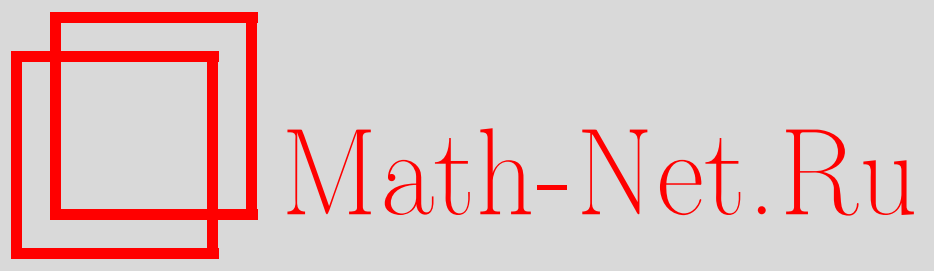

С. Н. Лакаев, А. М. Халхужаев, О спектре двухчастичного оператора Шредингера на решетке, ТМФ, 2008, том 155, номер 2, 287-300

DOI: https://doi.org/10.4213/tmf6211

Использование Общероссийского математического портала Math-Net.Ru подразумевает, что вы прочитали и согласны с пользовательским соглашением http://www. mathnet.ru/rus/agreement

Параметры загрузки:

IP : 54.80 .97 .219

26 апреля 2023 г., 15:13:18

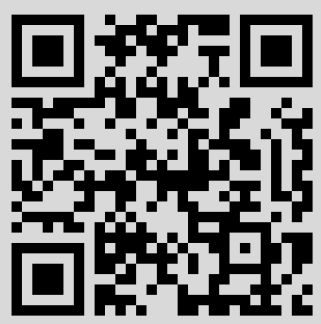




\title{
ФИЗИКА
}

Том 155, № 2

май, 2008

2008 г.

\author{
С. Н. Лакаев*, А. М. Халхужаев ${ }^{\dagger}$
}

\section{О СПЕКТРЕ ДВУХЧАСТИЧНОГО ОПЕРАТОРА ШРЕДИНГЕРА НА РЕШЕТКЕ}

\begin{abstract}
Рассматривается семейство двухчастичных дискретных операторов Шредингера $H(k)$, ассоциированных с гамильтонианом системы двух фермионов на $\nu$-мерной решетке $\mathbb{Z}^{\nu}, \nu \geqslant 1$, где $k \in \mathbb{T}^{\nu} \equiv(-\pi, \pi]^{\nu}$ - двухчастичный квазиимпульс. Доказано, что для любой размерности $\nu=1,2, \ldots$ оператор $H(k)$, $k \in \mathbb{T}^{\nu}, k \neq 0$, имеет собственное значение, лежащее левее существенного спектра, если оператор $H(0)$ имеет виртуальный уровень $(\nu=1,2)$ или собственное значение $(\nu \geqslant 3)$ на дне существенного спектра (двухчастичного континуума).
\end{abstract}

Ключевые слова: спектральные свойства, двухчастичный дискретный оператор Шредингера, принцип Бирмана-Швингера, виртуальный уровень, собственное значение.

\section{1. ВВЕДЕНИЕ}

Основной целью настоящей работы является доказательство существования собственных значений двухчастичных дискретных операторов Шредингера $H(k)$, ассоциированных с гамильтонианом системы двух фермионов на $\nu$-мерной решетке $\mathbb{Z}^{\nu}$, $\nu \geqslant 1$, при всех ненулевых значениях квазиимпульса $k \in \mathbb{T}^{\nu}$ и при условии, что оператор $H(0)$ имеет виртуальный уровень $(\nu=1,2)$ или собственное значение $(\nu \geqslant 3)$ на дне существенного спектра (двухчастичного континуума).

Спектральные свойства двухчастичных операторов Шредингера $H_{\mu}(k)=H_{0}(k)-$ $\mu V, k \in \mathbb{T}^{\nu}$, ассоциированных с гамильтонианом системы двух частиц на $\nu$-мерной решетке $\mathbb{Z}^{\nu}, \nu \geqslant 1$, и взаимодействующих с помощью короткодействующих потенциалов $\mu V$, изучены в работах [1]-[5]. В работах [1], [4], [5] рассматриваются гамильтонианы систем двух частиц, взаимодействующих с помощью парных контактных потенциалов притяжения. Установлено, что соответствующий оператор Шредингера $H_{\mu}(k)$ либо имеет единственное собственное значение, либо не имеет собственных значений в зависимости от значений энергии взаимодействия $\mu>0$ и квазиимпульса $k \in \mathbb{T}^{3}$.

\footnotetext{
* Самаркандский государственный университет, Самарканд, Узбекистан. E-mail: slakaev@mail.ru

${ }^{\dagger}$ Комплексный научно-исследовательский институт региональных проблем Самаркандского отделения АН РУз, Самарканд, Узбекистан. E-mail: ahmad_x@mail.ru
} 
В [2] доказано существование собственных значений двухчастичных операторов Шредингера $H(k)$, ассоциированных с гамильтонианом системы двух произвольных частиц на $\nu$-мерной решетке $\mathbb{Z}^{\nu}, \nu \geqslant 3$, при всех ненулевых значениях квазиимпульса $k \in \mathbb{T}^{\nu}$ и при условии, что оператор $H(0)$ имеет либо виртуальный уровень $(\nu=3,4)$, либо собственное значение $(\nu \geqslant 5)$ на дне существенного спектра (двухчастичного континуума).

Следует отметить следующее отличие: в случае бозонов дно существенного спектра является виртуальным уровнем (если размерность решетки $\nu=3,4$ ) и собственным значением при $\nu \geqslant 5$ оператора Шредингера $H(0)$. В настоящей статье для случая системы фермионов дно существенного спектра является виртуальным уровнем оператора $H(0)$ при $\nu=1,2$ (см. определение в разделе 4) и его собственным значением при $\nu \geqslant 3$.

Кроме того, в работе показано, что для любого $k \in \mathbb{T}^{\nu}$ оператор $H(k)$ может иметь лишь конечное число собственных значений, лежащих вне существенного спектра.

В настоящей работе через $C, C_{1}$ и $C_{2}$ обозначены положительные числа, принимающие различные значения; $\mathcal{U}_{\delta}(0)=\left\{K \in \mathbb{T}^{\nu}:|K|<\delta\right\}$ означает достаточно малую $\delta$-окрестность нуля. Через $\mathcal{B}\left(\theta, \mathcal{U}_{\delta}(0)\right), 0<\theta \leqslant 1$, обозначено банахово пространство непрерывных по Гёльдеру функций на $\mathcal{U}_{\delta}(0)$ с показателем $\theta$, полученное пополнением пространства гладких функций на $\mathcal{U}_{\delta}(0)$ относительно нормы

$$
\begin{gathered}
\|f\|_{\theta}=\sup _{k, \ell \in \mathbb{T}^{\nu}, \ell \neq 0}\left[|f(k)|+|\ell|^{-\theta}|f(k+\ell)-f(k)|\right] . \\
\text { 2. КООРДИНАТНОЕ И ИМПУЛЬСНОЕ } \\
\text { ПРЕДСТАВЛЕНИЯ ГАМИЛЬТОНИАНА СИСТЕМЫ } \\
\text { ДВУХ ЧАСТИЦ (ФЕРМИОНОВ) НА РЕШЕТКЕ }
\end{gathered}
$$

\section{2. КООРДИНАТНОЕ И ИМПУЛЬСНОЕ ПРЕДСТАВЛЕНИЯ ГАМИЛЬТОНИАНА СИСТЕМЫ ДВУХ ЧАСТИЦ (ФЕРМИОНОВ) НА РЕШЕТКЕ}

Пусть $\mathbb{Z}^{\nu}-\nu$-мерная целочисленная решетка и $\left(\mathbb{Z}^{\nu}\right)^{2}$ - декартово произведение $\mathbb{Z}^{\nu}$. Обозначим через $\ell_{2}\left(\left(\mathbb{Z}^{\nu}\right)^{2}\right)$ гильбертово пространство квадратично-суммируемых функций, определенных на $\left(\mathbb{Z}^{\nu}\right)^{2}$, и через $\ell_{2}^{\mathrm{a}}\left(\left(\mathbb{Z}^{\nu}\right)^{2}\right) \subset \ell_{2}\left(\left(\mathbb{Z}^{\nu}\right)^{2}\right)$ - подпространство, состоящее из антисимметричных функций.

Свободный гамильтониан $\widehat{H}_{0}$ рассматриваемой нами системы двух одинаковых частиц (фермионов) в координатном представлении определяется оператором, действующим в гильбертовом пространстве $\ell_{2}^{\mathrm{a}}\left(\left(\mathbb{Z}^{\nu}\right)^{2}\right)$, по формуле

$$
\widehat{H}_{0}=\frac{1}{2 m} \Delta_{x_{1}}+\frac{1}{2 m} \Delta_{x_{2}}
$$

где $\Delta_{x_{1}}=\Delta \otimes I$ и $\Delta_{x_{2}}=I \otimes \Delta$, а $m>0$ обозначает массу фермиона. Решетчатый лапласиан $\Delta$ есть разностный оператор, описывающий перенос частицы с каждого узла на соседний узел, т.е.

$$
(\Delta \hat{\psi})(x)=\sum_{|s|=1}[\hat{\psi}(x)-\hat{\psi}(x+s)], \quad \hat{\psi} \in \ell_{2}\left(\mathbb{Z}^{\nu}\right) .
$$

Гамильтониан системы двух фермионов (двухчастичный гамильтониан) $\widehat{H}$ описывает взаимодействие двух частиц в гильбертовом пространстве $\ell_{2}^{\mathrm{a}}\left(\left(\mathbb{Z}^{\nu}\right)^{2}\right)$ по формуле 
$\widehat{H}=\widehat{H}_{0}-\widehat{V}$, где $\widehat{V}$ - оператор умножения на вещественнозначную функцию:

$$
(\widehat{V} \hat{\psi})\left(x_{1}, x_{2}\right)=\widehat{V}\left(x_{1}-x_{2}\right) \hat{\psi}\left(x_{1}, x_{2}\right), \quad \hat{\psi} \in \ell_{2}^{\mathrm{a}}\left(\left(\mathbb{Z}^{\nu}\right)^{2}\right)
$$

Основное уСловиЕ. Предположим, что функиия $\widehat{V}(s) \geqslant 0$ является четной на $\mathbb{Z}^{\nu}$ и удовлетворяет условиям

$$
\begin{array}{rlll}
\lim _{|s| \rightarrow \infty}|s|^{2+\xi} \widehat{V}(s)=0 & \text { npu } & \nu=1 \quad \text { uлu } \quad \nu=2, \\
\lim _{|s| \rightarrow \infty}|s|^{3+1 / 2+\xi} \widehat{V}(s)=0 & \text { npu } & \nu=3, \\
\lim _{|s| \rightarrow \infty}|s|^{\nu+\xi} \widehat{V}(s)=0 & \text { npu } & \nu \geqslant 4,
\end{array}
$$

где $\xi>0$ - некоторое положительное число.

При выполнении основного условия двухчастичный гамильтониан $\widehat{H}$ является ограниченным самосопряженным оператором в $\ell_{2}^{\mathrm{a}}\left(\left(\mathbb{Z}^{\nu}\right)^{2}\right)$.

Пусть $\mathbb{T}^{\nu}-\nu$-мерный тор, т.е. куб $(-\pi, \pi]^{\nu}$ с соответствующим отождествлением противоположных граней. Он рассматривается как абелева группа, в которой операции сложения и умножения на вещественное число введены как операции сложения и умножения на вещественное число в $\mathbb{R}^{\nu}$ по модулю $(2 \pi \mathbb{Z})^{\nu}$.

Пусть $L_{2}\left(\left(\mathbb{T}^{\nu}\right)^{2}\right)$ - гильбертово пространство квадратично-интегрируемых функций, определенных на $\left(\mathbb{T}^{\nu}\right)^{2}, L_{2}^{\mathrm{a}}\left(\left(\mathbb{T}^{\nu}\right)^{2}\right) \subset L_{2}\left(\left(\mathbb{T}^{\nu}\right)^{2}\right)$ - подпространство антисимметричных функций. Пусть $\mathcal{F}: L_{2}\left(\left(\mathbb{T}^{\nu}\right)^{2}\right) \rightarrow \ell_{2}\left(\left(\mathbb{Z}^{\nu}\right)^{2}\right)$ - стандартное преобразование Фурье. Обозначим через $\widehat{\mathcal{F}}: L_{2}^{\mathrm{a}}\left(\left(\mathbb{T}^{\nu}\right)^{2}\right) \rightarrow \ell_{2}^{\mathrm{a}}\left(\left(\mathbb{Z}^{\nu}\right)^{2}\right)$ сужение $\mathcal{F}$ на $L_{2}^{\mathrm{a}}\left(\left(\mathbb{T}^{\nu}\right)^{2}\right)$.

Двухчастичный гамильтониан в импульсном представлении определяется как ограниченный самосопряженный оператор $H$, действующий в $L_{2}^{\mathrm{a}}\left(\left(\mathbb{T}^{\nu}\right)^{2}\right)$,

$$
H=\widehat{\mathcal{F}}^{-1} \widehat{H} \widehat{\mathcal{F}} \equiv H_{0}-V .
$$

Двухчастичный свободный гамильтониан $H_{0}$ является оператором умножения на функцию:

$$
\left(H_{0} f\right)\left(k_{1}, k_{2}\right)=\left(\frac{1}{m} \varepsilon\left(k_{1}\right)+\frac{1}{m} \varepsilon\left(k_{2}\right)\right) f\left(k_{1}, k_{2}\right), \quad f \in L_{2}^{\mathrm{a}}\left(\left(\mathbb{T}^{\nu}\right)^{2}\right),
$$

где

$$
\varepsilon(k)=\sum_{j=1}^{\nu}\left(1-\cos k^{(j)}\right), \quad k=\left(k^{(1)}, k^{(2)}, \ldots, k^{(\nu)}\right) \in \mathbb{T}^{\nu} .
$$

При выполнении основного условия возмущение $V$ определяется как ограниченный самосопряженный оператор, действующий в $L_{2}^{\mathrm{a}}\left(\left(\mathbb{T}^{\nu}\right)^{2}\right)$ по формуле

$$
(V f)\left(k_{1}, k_{2}\right)=(2 \pi)^{-\nu / 2} \int_{\left(\mathbb{T}^{\nu}\right)^{2}} V\left(k_{1}-k_{1}^{\prime}\right) \delta\left(k_{1}+k_{2}-k_{1}^{\prime}-k_{2}^{\prime}\right) f\left(k_{1}^{\prime}, k_{2}^{\prime}\right) d k_{1}^{\prime} d k_{2}^{\prime} .
$$

Здесь $\delta(k)$ обозначает дельта-функцию Дирака на $\mathbb{T}^{\nu}$, функция $V$ есть ряд Фурье

$$
V(k)=(2 \pi)^{-\nu / 2} \sum_{s \in \mathbb{Z}^{\nu}} \widehat{V}(s) e^{i(k, s)},
$$

4 Теоретическая и математическая физика, т. 155, № 2, 2008 г. 
где

$$
(k, s)=\sum_{j=1}^{\nu} k^{(j)} s^{(j)}, \quad k=\left(k^{(1)}, \ldots, k^{(\nu)}\right) \in \mathbb{T}^{\nu}, \quad s=\left(s^{(1)}, \ldots, s^{(\nu)}\right) \in \mathbb{Z}^{\nu} .
$$

Обозначим через $\widehat{U}_{s}, s \in \mathbb{Z}^{\nu}$, унитарный оператор, действующий в $\ell_{2}^{\mathrm{a}}\left(\left(\mathbb{Z}^{\nu}\right)^{2}\right)$ по формуле

$$
\left(\widehat{U}_{s} f\right)\left(n_{1}, n_{2}\right)=f\left(n_{1}+s, n_{2}+s\right), \quad f \in \ell_{2}^{\mathrm{a}}\left(\left(\mathbb{Z}^{\nu}\right)^{2}\right), \quad s \in \mathbb{Z}^{\nu}
$$

Так как для любых $s, p \in \mathbb{Z}^{\nu}$ имеет место равенство

$$
\widehat{U}_{s+p}=\widehat{U}_{s} \widehat{U}_{p}, \quad s, p \in \mathbb{Z}^{\nu},
$$

группа $\left\{\widehat{U}_{s}, s \in \mathbb{Z}^{\nu}\right\}$ образует унитарное представление абелевой группы $\mathbb{Z}^{\nu}$ в гильбертовом пространстве $\ell_{2}^{\mathrm{a}}\left(\left(\mathbb{Z}^{\nu}\right)^{2}\right)$.

После преобразования Фурье оператор сдвига $\widehat{U}_{s}$ переходит в унитарный оператор $U_{s}=\widehat{\mathscr{F}}^{-1} \widehat{U}_{s} \widehat{\mathcal{F}}, s \in \mathbb{Z}^{\nu}$, действующий как оператор умножения на функцию:

$$
\left(U_{s} f\right)\left(k_{1}, k_{2}\right)=e^{-i\left(s, k_{1}+k_{2}\right)} f\left(k_{1}, k_{2}\right), \quad f \in L_{2}^{\mathrm{a}}\left(\left(\mathbb{T}^{\nu}\right)^{2}\right) .
$$

Для любого $k \in \mathbb{T}^{\nu}$ обозначим

$$
\mathbb{F}_{k}=\left\{\left(k_{1}, k-k_{1}\right) \in\left(\mathbb{T}^{\nu}\right)^{2}: k_{1} \in \mathbb{T}^{\nu}, k-k_{1} \in \mathbb{T}^{\nu}\right\}
$$

Заметим, что $\mathbb{F}_{k}$ является $\nu$-мерным многообразием, изоморфным к $\mathbb{T}^{\nu}$. Действительно, рассмотрим отображение

$$
\pi:\left(\mathbb{T}^{\nu}\right)^{2} \rightarrow \mathbb{T}^{\nu}, \quad \pi\left(\left(k_{1}, k_{2}\right)\right)=q, \quad q=\frac{k_{1}+k_{2}}{2}-k_{2}
$$

Обозначим через $\pi_{k}, k \in \mathbb{T}^{\nu}$, сужение отображения $\pi$ на $\mathbb{F}_{k} \subset\left(\mathbb{T}^{\nu}\right)^{2}$, т.е.

$$
\pi_{k}=\left.\pi\right|_{\mathbb{F}_{k}}
$$

Отображение $\pi_{k}, k \in \mathbb{T}^{\nu}$, биективно отображает $\mathbb{F}_{k} \subset\left(\mathbb{T}^{\nu}\right)^{2}, k \in \mathbb{T}^{\nu}$, на $\mathbb{T}^{\nu}$ с обратным отображением

$$
\pi_{k}^{-1}(q)=\left(\frac{1}{2} k+q, \frac{1}{2} k-q\right)
$$

\section{3. РАЗЛОЖЕНИЕ В ПРЯМОЙ ОПЕРАТОРНЫЙ ИНТЕГРАЛ}

Пространство $L_{2}^{\mathrm{a}}\left(\left(\mathbb{T}^{\nu}\right)^{2}\right)$ разлагается в прямой интеграл

$$
L_{2}^{\mathrm{a}}\left(\left(\mathbb{T}^{\nu}\right)^{2}\right)=\int_{k \in \mathbb{T}^{\nu}} \oplus L_{2}^{\mathrm{a}}\left(\mathbb{F}_{k}\right) d k
$$

где $L_{2}^{\mathrm{a}}\left(\mathbb{F}_{k}\right)$ - пространство квадратично-интегрируемых и антисимметричных функций, определенных на $\mathbb{F}_{k}$. 
Соответственно унитарное отображение $U_{s}, s \in \mathbb{Z}^{\nu}$, разлагается в прямой интеграл

$$
U_{s}=\int_{k \in \mathbb{T}^{\nu}} \oplus U_{s}(k) d k,
$$

где

$$
U_{s}(k)=e^{-i(s, k)} I_{L_{2}^{\mathrm{a}}\left(\mathbb{F}_{k}\right)},
$$

а через $I_{L_{2}^{\mathrm{a}}\left(\mathbb{F}_{k}\right)}$ обозначен единичный оператор в пространстве $L_{2}^{\mathrm{a}}\left(\mathbb{F}_{k}\right)$.

Гамильтониан $\widehat{H}$ коммутирует с группой операторов $\widehat{U}_{s}, s \in \mathbb{Z}^{\nu}$, т.е. $\widehat{U}_{s} \widehat{H}=\widehat{H} \widehat{U}_{s}$, $s \in \mathbb{Z}^{\nu}$. Соответственно гамильтониан $H$ коммутирует с группой $U_{s}, s \in \mathbb{Z}^{\nu}$, и поэтому оператор $H$ разлагается в прямой интеграл

$$
H=\int_{k \in \mathbb{T}^{\nu}} \oplus \widetilde{H}(k) d k .
$$

Слойный оператор $\widetilde{H}(k), k \in \mathbb{T}^{\nu}$, действует в $L_{2}^{\mathrm{a}}\left(\mathbb{F}_{k}\right)$ по формуле

$$
\begin{aligned}
&(\widetilde{H}(k) f)(q, k-q)=\left[\frac{1}{m} \varepsilon(q)+\frac{1}{m} \varepsilon(k-q)\right] f(q, k-q)- \\
&-(2 \pi)^{-\nu / 2} \int_{\mathbb{T}^{\nu}} V(q-s) f(s, k-s) d s .
\end{aligned}
$$

Рассмотрим оператор $H(k), k \in \mathbb{T}^{\nu}$, действующий в гильбертовом пространстве $L_{2}^{\mathrm{o}}\left(\mathbb{T}^{\nu}\right)=\left\{f \in L_{2}\left(\mathbb{T}^{\nu}\right): f(-q)=-f(q)\right\}$ по формуле

$$
H(k)=H_{0}(k)-V .
$$

Невозмущенный оператор $H_{0}(k)$ есть оператор умножения на функцию $E_{k}(q)$ в пространстве $L_{2}^{\mathrm{o}}\left(\mathbb{T}^{\nu}\right)$, т.е.

$$
\left(H_{0}(k) f\right)(q)=E_{k}(q) f(q), \quad f \in L_{2}^{\mathrm{o}}\left(\mathbb{T}^{\nu}\right),
$$

где

$$
E_{k}(q)=\frac{1}{m} \varepsilon\left(\frac{1}{2} k+q\right)+\frac{1}{m} \varepsilon\left(\frac{1}{2} k-q\right) .
$$

Оператор взаимодействия (оператор возмущения) $V$ действует в гильбертовом пространстве $L_{2}^{\mathrm{o}}\left(\mathbb{T}^{\nu}\right)$ по формуле

$$
(V f)(q)=(2 \pi)^{-\nu / 2} \int_{\mathbb{T}^{\nu}} V(q-t) f(t) d t, \quad f \in L_{2}^{\mathrm{o}}\left(\mathbb{T}^{\nu}\right) .
$$

Слойный оператор $\widetilde{H}(k), k \in \mathbb{T}^{\nu}$, унитарно эквивалентен оператору $H(k), k \in \mathbb{T}^{\nu}$. Эта эквивалентность осуществляется с помощью унитарного оператора

$$
u_{k}: L_{2}^{\mathrm{a}}\left(\mathbb{F}_{k}\right) \rightarrow L_{2}^{\mathrm{o}}\left(\mathbb{T}^{\nu}\right), \quad k \in \mathbb{T}^{\nu}, \quad u_{k} g=g \circ \pi_{k}^{-1},
$$

где отображение $\pi_{k}$ определено в (2.3). 


\section{4. ФОРМУЛИРОВКА ОСНОВНЫХ РЕЗУЛЬТАТОВ}

При выполнении основного условия возмущение $V$ оператора $H_{0}(k)$ принадлежит классу операторов со следом. Следовательно, из теоремы Вейля о существенном спектре (см. [6]) существенный спектр $\sigma_{\text {ess }}(H(k))$ оператора $H(k)$, определенного в $(3.1)$, совпадает со спектром оператора $H_{0}(k)$. Так как $H_{0}(k)$ есть оператор умножения на функцию, то

$$
\sigma_{\mathrm{ess}}(H(k))=[m(k), M(k)]
$$

где

$$
m(k)=\min _{q \in \mathbb{T}^{\nu}} E_{k}(q), \quad M(k)=\max _{q \in \mathbb{T}^{\nu}} E_{k}(q) .
$$

ЗАмечаниЕ 1. При $k=(\pi, \pi, \ldots, \pi) \in \mathbb{T}^{\nu}$ существенный спектр оператора $H(k)$ вырождается, т.е. превращается в точку, и поэтому мы не можем утверждать, что $\sigma_{\text {ess }}(H(k))$ является абсолютно непрерывным при любом $k \in \mathbb{T}^{\nu}$.

В силу основного условия оператор $\widehat{V}$ является оператором умножения на положительную функцию $\widehat{V}(s)$, поэтому положительный корень $\widehat{V}^{1 / 2}$ является оператором умножения на положительную функцию $\widehat{V}^{1 / 2}(s)$. Следовательно, положительный корень $V^{1 / 2}$ оператора $V$ имеет вид

$$
\left(V^{1 / 2} f\right)(p)=(2 \pi)^{-\nu / 2} \int_{\mathbb{T}^{\nu}} V^{1 / 2}\left(p-p^{\prime}\right) f\left(p^{\prime}\right) d p^{\prime},
$$

где ядро $V^{1 / 2}(p)$ есть обратное преобразование Фурье функции $\widehat{V}^{1 / 2}(s)$, т.е.

$$
V^{1 / 2}(p)=\frac{1}{(2 \pi)^{\nu / 2}} \sum_{s \in \mathbb{Z}^{\nu}} \widehat{V}^{1 / 2}(s) e^{i(p, s)} .
$$

Для каждого $k \in(-\pi, \pi)^{\nu}$ и $z \leqslant m(k)$ (и для каждого $k \in \mathbb{T}^{\nu} \backslash(-\pi, \pi)^{\nu}$ и $z<m(k)$ ) определим интегральные операторы $G(k, z)$ и $G_{1 / 2}(k, z)$ соответственно с ядрами

$$
\begin{aligned}
G(k, z ; p, s) & =\frac{1}{(2 \pi)^{\nu}} \int_{\mathbb{T}^{\nu}} \frac{V^{1 / 2}(p-t) V^{1 / 2}(t-s) d t}{E_{k}(t)-z}, \\
G_{1 / 2}(k, z ; p, s) & =\frac{1}{(2 \pi)^{\nu / 2}} \frac{V^{1 / 2}(p-s)}{\left(E_{k}(p)-z\right)^{1 / 2}} .
\end{aligned}
$$

Отметим, что для любого $z<m(k)$ имеют место следующие равенства: $G(k, z)=$ $V^{1 / 2} R_{0}(k, z) V^{1 / 2}$ и $G_{1 / 2}(k, z)=R_{0}^{1 / 2}(k, z) V^{1 / 2}$, где $R_{0}(k, z)$ - резольвента оператоpa $H_{0}(k)$.

ОПРЕДЕЛЕНИЕ. Пусть $\nu=1$ или $\nu=2$ и выполняется основное условие. Предположим, что уравнение $G(0,0) \psi=\psi$ имеет ненулевое решение $\psi \in L_{2}^{\mathrm{o}}\left(\mathbb{T}^{\nu}\right)$. Будем говорить, что оператор $H(0)$ имеет виртуальный уровень в нуле, если

$$
\frac{\left(V^{1 / 2} \psi\right)(p)}{E_{0}(p)} \notin L_{2}^{\mathrm{o}}\left(\mathbb{T}^{\nu}\right)
$$

(см. также ниже лемму 3). 
Дальнейшие результаты опираются только на уравнение $G(0,0) \psi=\psi$. Однако функция

$$
\frac{\left(V^{1 / 2} \psi\right)(p)}{E_{0}(p)}
$$

может как принадлежать пространству $L_{2}^{\mathrm{o}}\left(\mathbb{T}^{\nu}\right)$, так и не принадлежать ему. В первом случае нуль является собственным значением оператора $H(0)$, а во втором случае естественно говорить о виртуальном уровне.

Предположим, что выполняется основное условие. Тогда справедливы следующие теоремы.

ТЕОРема 1. Пусть $\nu=1$ или $\nu=2$. Если число $\lambda=1$ является собственным значением оператора $G(0,0)$, то для любого $k \neq 0$ оператор $H(k)$ имеет собственное значение, лежащее левее существенного спектра.

ТеОРема 2. Пусть $\nu \geqslant 3$. Если число $z=0$ является $n$-кратным собственным значением оператора $H(0)$, то для любого $k \neq 0$ оператор $H(k)$ имеет не менее $n$ собственных значений, лежащих левее существенного спектра.

Теорема 3. Для любъх $k \in(-\pi, \pi)^{\nu} u \nu \in \mathbb{N}$ оператор $H(k)$ может иметь лищь конечное число собственных значений, лежащих левее существенного спекmpa.

ЗАмечАниЕ 2. Так как возмущенный оператор $V$ положителен, $H(k)$ не имеет собственных значений, лежащих правее $M(k)$.

ЗАмЕЧАНИЕ 3. Множество операторов, удовлетворяющих условиям теорем 1 и 2 , не пусто (см. пример в разделе 6).

\section{5. ПРИНЦИП БИРМАНА-ШВИНГЕРА}

Лемма 1. Оператор $G(k, z)$ действует в $L_{2}^{\mathrm{o}}\left(\mathbb{T}^{\nu}\right)$, положителен, непрерывен по $z \leqslant m(k)$ и принадлежит классу $\Sigma_{1}$.

ДокАЗАТЕЛЬство. Функцию $E_{k}(q)$, определенную по формуле $(3.3)$, представим в виде

$$
E_{k}(q)=\frac{2}{m} \sum_{i=1}^{\nu}\left(1-\cos \frac{k^{(i)}}{2} \cos q^{(i)}\right) .
$$

Из четности функций $E_{k}(q)$ и $V^{1 / 2}(q)$ на $\mathbb{T}^{\nu}$ следует, что

$$
G_{1 / 2}(k, z ;-p,-s)=G_{1 / 2}(k, z ; p, s) .
$$

Поэтому пространство $L_{2}^{\mathrm{o}}\left(\mathbb{T}^{\nu}\right)$ инвариантно относительно оператора $G_{1 / 2}(k, z)$.

Напомним (см. [6, теорема VI.22]), что оператор $A$ принадлежит классу операторов Гильберта-Шмидта тогда и только тогда, когда $\left\{\left\|A \varphi_{n}\right\|\right\} \in \ell_{2}\left(\mathbb{Z}^{\nu}\right)$ для некоторого ортонормированного базиса $\varphi_{n}$.

Пусть

$$
\left\{\varphi_{n}(p)=\frac{\sqrt{2}}{(2 \pi)^{\nu}} \sin (n, p), n \in \mathbb{Z}^{\nu}\right\}
$$


- ортонормированный базис в $L_{2}^{\mathrm{o}}\left(\mathbb{T}^{\nu}\right)$. Тогда

$$
\left(G_{1 / 2}(k, z) \varphi_{n}\right)(p)=\frac{\widehat{V}^{1 / 2}(n) \varphi_{n}(p)}{\sqrt{E_{k}(p)-z}}, \quad z \leqslant m(k) .
$$

Имеем

$$
\left\|G_{1 / 2}(k, z) \varphi_{n}\right\|^{2}=\frac{2 \widehat{V}(n)}{(2 \pi)^{\nu}} \int_{\mathbb{T}^{\nu}} \frac{\sin ^{2}(n, q) d q}{E_{k}(q)-z}, \quad n \in \mathbb{Z}^{\nu} .
$$

Пусть $\nu=1$ или $\nu=2$. Так как для каждого $0<\zeta \leqslant 1$ и $q \in \mathbb{T}^{\nu}, n \in \mathbb{Z}^{\nu}$, имеет место неравенство $\sin (n, q) \leqslant|q|^{\zeta}|n|^{\zeta}$, интеграл в правой части равенства (5.1) удовлетворяет неравенству

$$
\int_{\mathbb{T}^{\nu}} \frac{\sin ^{2}(n, q) d q}{E_{k}(q)-z} \leqslant \int_{\mathbb{T}^{\nu}} \frac{|n|^{2 \zeta}|q|^{2 \zeta} d q}{\frac{2}{m} \sum_{i=1}^{\nu}\left(1-\cos \left(k^{(i)} / 2\right) \cos q^{(i)}\right)-z} .
$$

Знаменатель подынтегральной функции в правой части неравенства (5.2) имеет невырожденный минимум в нуле. Поэтому, переходя к сферическим системам координат, после вычислений получим неравенство

$$
\int_{\mathbb{T}^{\nu}} \frac{|q|^{2 \zeta} d q}{\frac{2}{m} \sum_{i=1}^{\nu}\left(1-\cos \left(k^{(i)} / 2\right) \cos q^{(i)}\right)-z} \leqslant C_{1} \int_{0}^{\delta} \frac{d t}{|t|^{2-2 \zeta-\nu+1}}+C_{2} .
$$

Интеграл правой части неравенства (5.3) сходится в одномерном случае при $\zeta>$ $1 / 2$ и в двумерном случае при $\zeta>0$. Учитывая соотношения (5.1)-(5.3), в силу основного условия для каждого $\xi>0$ получим, выбирая $1<2 \zeta<1+\xi$ при $\nu=1$ и $0<2 \zeta<\xi$ при $\nu=2$, что $\left\{\left\|G_{1 / 2}(k, z) \varphi_{n}\right\|\right\} \in \ell_{2}\left(\mathbb{Z}^{\nu}\right)$.

Пусть $\nu \geqslant 3$. Пользуясь неравенством $\sin (n, q) \leqslant 1$, оценим интеграл в правой части равенства (5.1) сверху интегралом

$$
(2 \pi)^{-\nu} \int_{\mathbb{T}^{\nu}} \frac{d q}{E_{k}(q)-z}, \quad z \leqslant m(k) .
$$

Так как функция $E_{k}(q), k \in(-\pi, \pi)^{\nu}$, имеет единственный невырожденный минимум в $q=0$, этот интеграл сходится и его значение мы обозначим через $d(k, z)$. Имеем

$$
\left\|G_{1 / 2}(k, z) \varphi_{n}\right\|^{2} \leqslant C d(k, z) \widehat{V}(n), \quad n \in \mathbb{Z}^{\nu} .
$$

Пользуясь основным условием, получим, что $\left\{\left\|G_{1 / 2}(k, z) \varphi_{n}\right\|\right\} \in \ell_{2}\left(\mathbb{Z}^{\nu}\right)$. Поэтому $G_{1 / 2}(k, z)$ принадлежит классу операторов Гильберта-Шмидта.

Из монотонности интеграла Лебега вытекает, что $G_{1 / 2}(k, z)$ как операторнозначная функция является непрерывной слева по $z \leqslant m(k)$. Из равенства $G(k, z)=$ $\left(G_{1 / 2}(k, z)\right)^{*} G_{1 / 2}(k, z)$ вытекает, что оператор $G(k, z)$ также непрерывен слева по $z \leqslant m(k)$, положителен и $G(k, z) \in \Sigma_{1}$.

Для ограниченного самосопряженного оператора $A$, действующего в гильбертовом пространстве $\mathcal{H}$ и не имеющего существенного спектра правее (соответственно левее) точки $\mu \in \mathbb{R}$, определим $n_{+}(\mu, A)$ (соответственно $\left.n_{-}(\mu, A)\right)$ :

$$
\begin{aligned}
n_{+}(\mu, A) & =\sup \{\operatorname{dim} L: L \subset \mathcal{H} ;(A f, f)>\mu,\|f\|=1\}, \\
\left(n_{-}(\mu, A)\right. & =\sup \{\operatorname{dim} L: L \subset \mathcal{H} ;(A f, f)<\mu,\|f\|=1\}) .
\end{aligned}
$$


Число $n_{+}(\mu, A)$ (соответственно $\left.n_{-}(\mu, A)\right)$ совпадает с числом собственных значений оператора $A$, лежащих правее (соответственно левее) точки $\mu$.

Для оператора $H(k), k \in \mathbb{T}^{\nu}$, справедлив следующий принцип Бирмана-Швингера.

ЛЕмма 2. Для каждого $k \in(-\pi, \pi)^{\nu} u z \leqslant m(k)$ выполняется равенство

$$
n_{-}(z, H(k))=n_{+}(1, G(k, z)) .
$$

ДоказАТЕЛьство. Пусть $z<m(k)$. Сначала мы докажем неравенство

$$
n_{-}(z, H(k)) \leqslant n_{+}(1, G(k, z)) .
$$

Пусть $z_{1}(k) \leqslant z_{2}(k) \leqslant \cdots \leqslant z_{m}(k)<m(k)$ - собственные значения оператора $H(k)$. Обозначим через $L_{i}$ подпространство, порожденное собственными функциями, соответствующими собственным значениям $z_{i}(k), i=\overline{1, m}$. Для любого $u \in L_{m}$ имеем

$$
\left(\left(H_{0}(k)-z\right) u, u\right)<(V u, u) .
$$

Это неравенство выполняется тогда и только тогда, когда

$$
(y, y)<\left(R_{0}^{1 / 2}(k, z) V R_{0}^{1 / 2}(k, z) y, y\right)
$$

где $y=\left(H_{0}(k)-z\right)^{1 / 2} u$. Следовательно,

$$
n_{-}(z, H(k)) \leqslant n_{+}\left(1, R_{0}^{1 / 2}(k, z) V R_{0}^{1 / 2}(k, z)\right) .
$$

Для ограниченных операторов $A$ и $B$ каждое ненулевое собственное значение оператора $A B$ является собственным значением $B A$ с той же кратностью (см., например, [7]), поэтому имеем

$$
n_{+}\left(1, R_{0}^{1 / 2}(k, z) V R_{0}^{1 / 2}(k, z)\right)=n_{+}\left(1, V^{1 / 2} R_{0}(k, z) V^{1 / 2}\right) .
$$

Отсюда и из (5.6) получаем

$$
n_{-}(z, H(k)) \leqslant n_{+}(1, G(k, z)) .
$$

Заменяя аргументы, мы получим неравенство, обратное к (5.7), и, следовательно,

$$
n_{-}(z, H(k))=n_{+}(1, G(k, z)) .
$$

Осталось доказать равенство $n_{+}(1, G(k, m(k)))=n_{-}(m(k), H(k))$. Так как оператор $G(k, m(k))$ компактен, число $n_{+}(1, G(k, m(k)))$ конечно. Для любых $\psi \in L_{2}^{\circ}\left(\mathbb{T}^{\nu}\right)$ и $z<m(k)$ имеем

$$
\begin{aligned}
(G(k, z) \psi, \psi) & =\left(V^{1 / 2} R_{0}(k, z) V^{1 / 2} \psi, \psi\right)=\left(R_{0}(k, z) V^{1 / 2} \psi, V^{1 / 2} \psi\right)= \\
& =\int_{\mathbb{T}^{\nu}} \frac{\left|\left(V^{1 / 2} \psi\right)(p)\right|^{2} d p}{E_{k}(p)-z} \leqslant \int_{\mathbb{T}^{\nu}} \frac{\left|\left(V^{1 / 2} \psi\right)(p)\right|^{2} d p}{E_{k}(p)-m(k)}=(G(k, m(k)) \psi, \psi) .
\end{aligned}
$$


Следовательно,

$$
n_{+}(1, G(k, z)) \leqslant n_{+}(1, G(k, m(k))) .
$$

Пусть $\lambda_{1}(k) \geqslant \lambda_{2}(k) \geqslant \cdots \geqslant \lambda_{n}(k)>1$ - собственные значения оператора $G(k, m(k))$ и $H_{i}$ - подпространство, порожденное собственными функциями, соответствующими собственным значениям $\lambda_{i}(k), i=\overline{1, n}$.

Для любого $\varphi \in H_{n}$ имеют место неравенства

$$
\begin{aligned}
(G(k, z) \varphi, \varphi) & =(G(k, m(k)) \varphi, \varphi)+(G(k, z)-G(k, m(k)) \varphi, \varphi)> \\
& >\left(\lambda_{n}(k) \varphi, \varphi\right)+\left(\left(1-\lambda_{n}(k)\right) \varphi, \varphi\right)=(\varphi, \varphi), \\
& \|G(k, m(k))-G(k, z)\|<\lambda_{n}(k)-1 .
\end{aligned}
$$

Отсюда получаем

$$
n_{+}(1, G(k, z)) \geqslant \operatorname{dim} H_{n}=n_{+}(1, G(k, m(k))) .
$$

Из неравенств (5.9), (5.10) следует, что

$$
n_{+}(1, G(k, z))=n_{+}(1, G(k, m(k)))
$$

для достаточно малых $m(k)-z$. Существует $\delta>0$ такое, что

$$
n_{-}(z, H(k))=n_{-}(m(k), H(k)), \quad z \in(m(k)-\delta, m(k)] .
$$

Учитывая (5.8), (5.11), (5.12), получим равенство (5.4).

В следующей лемме описываются некоторые свойства функций, удовлетворяющих уравнению Бирмана-Швингера, и ассоциированных с ними собственных функций.

ЛЕмма 3. Пусть уравнение

$$
G(0,0) \psi=\psi
$$

имеет ненулевое решение $\psi \in L_{2}^{\mathrm{o}}\left(\mathbb{T}^{\nu}\right)$ u $\varphi(p)=\left(V^{1 / 2} \psi\right)(p)$. Тогда функция $f(p)=$ $\varphi(p) / E_{0}(p)$ удовлетворяет уравнению $H(0) f=0$. Функиия $\varphi(p)$ является нечетной и удовлетворяет следующим соотношениям:

$$
\begin{array}{llll}
\varphi(p) \in \mathcal{B}\left(\theta, \mathcal{U}_{\delta}(0)\right), & \frac{1}{2}<\theta<\min \left\{1, \frac{1+\xi}{2}\right\} & \text { npu } & \nu=1, \\
\varphi(p) \in \mathcal{B}\left(\theta, \mathcal{U}_{\delta}(0)\right), & 0<\theta<\min \left\{\frac{1}{2}, \frac{\xi}{2}\right\}, & \text { npu } & \nu=2, \\
\varphi(p) \in \mathcal{B}\left(\theta, \mathcal{U}_{\delta}(0)\right), & \frac{1}{2}<\theta<\min \left\{1, \frac{1}{2}+\xi\right\} & \text { npu } & \nu=3, \\
\varphi(p) \in \mathcal{B}\left(\theta, \mathcal{U}_{\delta}(0)\right), & 0<\theta<\min \{1, \xi\} & \text { npu } & \nu \geqslant 4,
\end{array}
$$

где $\xi>0$ определено в основном условии. 
ДокАзАТЕЛЬСтво. Доказательство нечетности функции $\varphi(p)$ на $\mathbb{T}^{\nu}$ непосредственно вытекает из четности функции $V^{1 / 2}(p)$ на $\mathbb{T}^{\nu}$.

Из свойств преобразования Фурье следует, что имеет место равенство

$$
V(p-s)=\frac{1}{(2 \pi)^{\nu / 2}} \int_{\mathbb{T}^{\nu}} V^{1 / 2}(p-t) V^{1 / 2}(t-s) d t .
$$

Так как $\psi \in L_{2}^{\mathrm{o}}\left(\mathbb{T}^{\nu}\right)$ есть решение уравнения (5.13), в силу (5.18) имеем, что

$$
\varphi(p)=\frac{1}{(2 \pi)^{\nu / 2}} \int_{\mathbb{T}^{\nu}} V^{1 / 2}\left(p-q^{\prime}\right) \psi\left(q^{\prime}\right) d q^{\prime}
$$

удовлетворяет уравнению

$$
\varphi(p)=\frac{1}{(2 \pi)^{\nu / 2}} \int_{\mathbb{T}^{\nu}} \frac{V(p-t) \varphi(t) d t}{E_{0}(t)} .
$$

Поэтому функция $f(p)=\varphi(p) / E_{0}(p)$ удовлетворяет уравнению $H(0) f=0$.

Пользуясь неравенством Коши-Буняковского и равенством (5.18), получаем

$$
\begin{aligned}
|\varphi(p+q)-\varphi(p)|^{2} & =\left|\frac{1}{(2 \pi)^{\nu / 2}} \int_{\mathbb{T}^{\nu}}\left(V^{1 / 2}(p+q-t)-V^{1 / 2}(p-t)\right) \psi(t) d t\right|^{2} \leqslant \\
& \leqslant \frac{1}{(2 \pi)^{\nu}} \int_{\mathbb{T}^{\nu}}\left|V^{1 / 2}(p+q-t)-V^{1 / 2}(p-t)\right|^{2} d t \int_{\mathbb{T}^{\nu}}|\psi(t)|^{2} d t= \\
& =\frac{2}{(2 \pi)^{\nu}}(V(0)-V(q))\|\psi\|^{2} .
\end{aligned}
$$

Пусть $\nu=1$. Из основного условия и теории рядов Фурье следует, что функция $V$ непрерывно дифференцируема и

$$
\frac{\partial V(q)}{\partial q} \in \mathcal{B}\left(\theta_{1}, \mathcal{U}_{\delta}(0)\right), \quad 0<\theta_{1}<\min \{1, \xi\} .
$$

Из четности функции $V$ на $\mathbb{T}^{1}$ вытекает равенство $\partial V(0) / \partial q=0$. По формуле Тейлора для малых $q \in \mathbb{T}^{1}$ имеем

$$
V(q)=V(0)+\widetilde{V}(q),
$$

где $|\widetilde{V}(q)| \leqslant C|q|^{1+\theta_{1}}$.

Отсюда, учитывая неравенство (5.19), получаем

$$
|\varphi(p+q)-\varphi(p)| \leqslant C|q|^{\theta}, \quad \frac{1}{2}<\theta<\min \left\{1, \frac{1+\xi}{2}\right\} .
$$

Пусть $\nu=2$. Так как для каждого $0<2 \theta \leqslant 1$ и $q \in \mathbb{T}^{2}, n \in \mathbb{Z}^{2}$ имеет место неравенство $\left|e^{i(q, n)}-1\right| \leqslant|q|^{2 \theta}|n|^{2 \theta}$, в силу основного условия имеем

$$
|V(q)-V(0)|=\left|\frac{1}{2 \pi} \sum_{s \in \mathbb{Z}^{2}} \widehat{V}(s)\left(e^{i(q, s)}-1\right)\right| \leqslant C \sum_{s \in \mathbb{Z}^{2}, s \neq 0} \frac{|q|^{2 \theta}|s|^{2 \theta}}{|s|^{2+\varepsilon}} .
$$


Ряд, стоящий в правой части неравенства (5.20), сходится при $0<2 \theta<\min \{1, \varepsilon\}$. Отсюда и из (5.19) вытекает соотношение (5.15).

Пусть $\nu \geqslant 3$. Имеем

$$
|\varphi(p+q)-\varphi(p)| \leqslant \frac{1}{(2 \pi)^{\nu / 2}} \sup _{t \in \mathbb{T}^{\nu}}|V(p+q-t)-V(p-t)| \int_{\mathbb{T}^{\nu}} \frac{|\varphi(t)| d t}{E_{0}(t)} .
$$

Так как функция $E_{0}(p)$ имеет невырожденный минимум в нуле, существуют числа $C>0$ и $\delta>0$ такие, что

$$
E_{0}(p) \geqslant C|p|^{2}, \quad p \in \mathcal{U}_{\delta}(0)
$$

Отсюда и из непрерывности функции $\varphi$ следует, что

$$
\int_{\mathbb{T}^{\nu}} \frac{|\varphi(t)| d t}{E_{0}(t)}<\infty
$$

В силу основного условия имеем

$$
\begin{aligned}
& |V(p+q-t)-V(p-t)| \leqslant C_{1}|q|^{\theta}, \quad \frac{1}{2}<\theta<\min \left\{1, \frac{1}{2}+\xi\right\} \quad \text { при } \quad \nu=3, \\
& |V(p+q-t)-V(p-t)| \leqslant C_{2}|q|^{\theta}, \quad 0<\theta<\min \{1, \xi\} \quad \text { при } \nu \geqslant 4 . \\
& \text { Учитывая (5.21), (5.23)-(5.25), получим соотношения (5.16), (5.17). }
\end{aligned}
$$

ЛЕмма 4. Пусть $\nu \geqslant 3$. Число $\lambda=1$ является собственным значением оператора $G(0,0)$ тогда и только тогда, когда число $z=0$ является собственным значением оператора $H(0)$.

ДоказАтельство. Необходимость. Пусть уравнение $G(0,0) \psi=\psi$ имеет ненулевое решение $\psi \in L_{2}^{\mathrm{o}}\left(\mathbb{T}^{\nu}\right)$. В силу утверждения леммы 3 функция

$$
f(p)=\frac{\left(V^{1 / 2} \psi\right)(p)}{E_{0}(p)}
$$

удовлетворяет уравнению $H(0) f=0$. Пользуясь неравенствами (5.16), (5.17), (5.22), получим, что для функции $f$, определенной в $(5.26)$, имеют место следующие соотношения:

$$
\begin{aligned}
& \int_{\mathbb{T}^{3}}|f(p)|^{2} d p=\int_{\mathbb{T}^{3}} \frac{|\varphi(p)|^{2}}{\left(E_{0}(p)\right)^{2}} d p \leqslant C_{1}+C_{2} \int_{0}^{\delta} \frac{d r}{r^{2-2 \theta}}<\infty, \quad \frac{1}{2}<\theta<\min \left\{1, \frac{1}{2}+\xi\right\}, \\
& \int_{\mathbb{T}^{\nu}}|f(p)|^{2} d p=\int_{\mathbb{T}^{\nu}} \frac{|\varphi(p)|^{2}}{\left(E_{0}(p)\right)^{2}} d p \leqslant C_{1}+C_{2} \int_{0}^{\delta} \frac{d r}{r^{5-\nu-2 \theta}}<\infty, \quad 0<\theta<\min \{1, \xi\} .
\end{aligned}
$$

Следовательно, $f \in L_{2}^{\mathrm{o}}\left(\mathbb{T}^{\nu}\right)$, т.е. число $z=0$ является собственным значением оператора $H(0)$.

Достаточность. Пусть число $z=0$ является собственным значением оператора $H(0)$, т.е. уравнение

$$
E_{0}(q) f(q)=\frac{1}{(2 \pi)^{\nu / 2}} \int_{\mathbb{T}^{\nu}} V(q-t) f(t) d t
$$


имеет ненулевое решение $f \in L_{2}^{\mathrm{o}}\left(\mathbb{T}^{\nu}\right)$.

Введем обозначение

$$
\psi(p)=\frac{1}{(2 \pi)^{\nu / 2}} \int_{\mathbb{T}^{\nu}} V^{1 / 2}\left(p-q^{\prime}\right) f\left(q^{\prime}\right) d q^{\prime} .
$$

Тогда из (5.27) получаем

$$
f(p)=\frac{(2 \pi)^{-\nu / 2}}{E_{0}(p)} \int_{\mathbb{T}^{\nu}} V^{1 / 2}\left(p-q^{\prime}\right) \psi\left(q^{\prime}\right) d q^{\prime} .
$$

Подставляя правую часть равенства (5.29) в (5.28), получим, что уравнение $\psi=$ $G(0,0) \psi$ имеет ненулевое решение $\psi \in L_{2}^{\circ}\left(\mathbb{T}^{\nu}\right)$, т.е. число $\lambda=1$ является собственным значением оператора $G(0,0)$.

\section{6. ДОКАЗАТЕЛЬСТВО ОСНОВНЫХ РЕЗУЛЬТАТОВ}

ДокАЗАТЕЛЬСтво теоремы 1. По определению уравнение $G(0,0) \psi=\psi$ имеет ненулевое решение $\psi \in L_{2}^{\mathrm{o}}\left(\mathbb{T}^{\nu}\right)$. Нормируем $\|\psi\|=1$. Тогда

$$
(\psi, \psi)=(G(0,0) \psi, \psi)=\int_{\mathbb{T}^{\nu}}\left(E_{0}(p)\right)^{-1}|\varphi(p)|^{2} d p=1
$$

Для любого ненулевого $k \in(-\pi, \pi)^{\nu}$ почти всюду имеет место соотношение $0<$ $E_{k}(p)-m(k)<E_{0}(p)$. Следовательно,

$$
1=\int_{\mathbb{T}^{\nu}} \frac{|\varphi(p)|^{2} d p}{E_{0}(p)}<\int_{\mathbb{T}^{\nu}} \frac{|\varphi(p)|^{2} d p}{E_{k}(p)-m(k)}=(G(k, m(k)) \psi, \psi) .
$$

Это означает, что $n_{+}(1, G(k, m(k))) \geqslant 1$. Из непрерывности оператора $G(k, z)$ по $z \leqslant m(k)$ следует, что существует $z_{0}<m(k)$ такое, что выполняется неравенство $\left(G\left(k, z_{0}\right) \psi, \psi\right)>1$. Согласно лемме 2 оператор $H(k)$ при $k \neq 0$ имеет собственное значение, лежащее левее существенного спектра. Теорема 1 доказана.

ДОКАЗАТЕЛЬСТво ТЕОРЕмЫ 2 . Пусть число $z=0$ является $n$-кратным собственным значением оператора $H(0)$. Для любого ненулевого $k \in(-\pi, \pi)^{\nu}$ почти всюду выполняется соотношение $0<E_{k}(p)-m(k)<E_{0}(p)$. Следовательно,

$$
((H(k)-m(k)) f, f)<(H(0) f, f), \quad f \in L_{2}^{\mathrm{o}}\left(\mathbb{T}^{\nu}\right), \quad f \neq 0
$$

Отсюда и из условия теоремы и принципа минимакса (см. [6]) мы заключаем, что оператор $H(k)$ имеет не менее $n$ собственных значений, лежащих левее существенного спектра.

ДокАЗАТЕЛЬство теоремы 3. Доказательство теоремы 3 непосредственно вытекает из положительности оператора $V$, компактности оператора $G(k, m(k))$, $k \in \mathbb{T}^{\nu}$, и утверждения леммы 2 . 
Пример. Приведем пример того, что число $z=0$ является собственным значением оператора $H_{\mu}(0)$ или оператор $H_{\mu}(0)$ имеет виртуальный уровень в нуле в зависимости от размерности решетки.

Рассмотрим оператор $H_{\mu}(k), k \in \mathbb{T}^{\nu}$, действующий в $L_{2}^{\mathrm{o}}\left(\mathbb{T}^{\nu}\right)$ по формуле

$$
H_{\mu}(k)=H_{0}(k)-\mu V .
$$

Здесь оператор $H_{0}(k)$ определен по формуле $(3.2)$, а $V$ действует в $L_{2}^{\mathrm{o}}\left(\mathbb{T}^{\nu}\right)$ согласно формуле

$$
(V f)(p)=\frac{1}{(2 \pi)^{\nu}} \int_{\mathbb{T}^{\nu}} \sum_{i=1}^{\nu} \sin p^{(i)} \sin t^{(i)} f(t) d t,
$$

$\mu>0$ - энергия взаимодействия двух частиц (фермионов).

Преобразование Фурье ядра оператора $V$ на $\mathbb{Z}^{\nu}$ имеет вид

$$
\widehat{V}(s)=\left\{\begin{array}{lll}
1 / 2 & \text { при } & |s|=1, \\
0 & \text { при } & |s| \neq 1 .
\end{array}\right.
$$

Нетривиальное решение уравнения $H_{\mu}(0) f=0$ имеет вид

$$
f(p)=\frac{\mu \sum_{i=1}^{\nu} \alpha_{i} \sin p^{(i)}}{E_{0}(p)},
$$

где $\alpha_{i}$ - ненулевые коэффиценты.

Так как функция $E_{0}(p)$ имеет единственный невырожденный минимум в нуле и $|\sin x| \leqslant|x|$, функция $f$, определенная в (6.1), принадлежит $L_{2}^{\mathrm{o}}\left(\mathbb{T}^{\nu}\right)$ при $\nu \geqslant 3$. Следовательно, нуль является собственным значением оператора $H_{\mu}(0)$ при $\nu \geqslant 3$ и его виртуальным уровнем при $\nu=1,2$.

\section{Список литературы}

[1] S. Albeverio, S. N. Lakaev, Z. I. Muminov, Ann. Inst. H. Poincaré Phys. Théor., 5:4 (2004), 743-772.

[2] S. Albeverio, S. N. Lakaev, K. A. Makarov, Z. I. Muminov, Comm. Math. Phys., 262:1 (2006), 91-115.

[3] G. M. Graf, D. Schenker, Ann. Inst. H. Poincaré Phys. Théor., 67:1 (1997), 91-107.

[4] С. Н. Лакаев, Функи. анализ и его прил., 27:3 (1993), 15-28.

[5] P. A. Faria da Veiga, L. Ioriatti, M. O'Carroll, Phys. Rev. E (3), 66:1 (2002), 6130.

[6] М. Рид, Б. Саймон, Методы современной математической физики. Т. 4. Анализ операторов, Мир, М., 1982.

[7] П. Р. Халмош, Гилъбертово пространство в задачах, Мир, М., 1970.

Поступила в редакцию 20.12.2005, после доработки 24.07.2007 